\title{
Zwei altuigurische Bekehrungstexte aus der manichäischen Missionsgeschichte
}

\author{
Peter Zieme (Berlin)
}

Gewidmet Werner Sundermann zu seinem 75. Geburtstag

Die Mission spielte für den Gründer der nach ihm benannten Lichtreligion eine herausragende Rolle. Mani selbst und seine Schüler und Nachfolger unternahmen ausgiebig Reisen mit dem Ziel der Verbreitung der Lehre. Zu bestimmten Zeiten war der Manichäismus dann auch von Nordafrika bis Südchina präsent und wirksam, seine letzten Spuren lassen sich bis ins 17 . Jh. in der Provinz Fujian nachweisen. Einmalig in der manichäischen Kirchengeschichte war die Annahme der Religion durch den Kagan Bögü, den Herrscher des Uigurischen Steppenimperiums mit der Hauptstadt Karabalgasun, in der Mitte des 8. Jh., ein Ereignis, das auch den Fortbestand der Manichäer in den Oasen Dunhuang und vor allem Turfan im 8. sowie darüber hinaus auch bis etwa zur Mitte des 11. Jh. sicherte. Die zentralasiatischen Quellen sind spärlich, umfangreicher als alttürkische Bezeugungen sind die mitteliranischen Fragmente, die in mehreren Arbeiten von WALTER B. HENNING, dann aber vor allem in grundlegenden und zusammenfassenden Studien von WERNER SUNDERMANN ediert und bewertet wurden. Die alttürkischen Fragmente, die diesem Bereich des manichäischen Schrifttums zugeordnet werden können, sind zum größten Teil bekannt und bearbeitet ${ }^{1}$. Hier sollen Reste von zwei unterschiedlichen Büchern vorgelegt werden, die noch nicht zur Gänze die ihnen zukommende Beachtung gefunden haben. Im ersteren Fall ließ sich für einen Passus eine genaue parthische Vorlage ermitteln.

\section{I.}

Fragmente auf der Rückseite einer chinesischen Buchrolle

Fünf Textreste auf den Rückseiten chinesischer buddhistischer Buchrollenfragmente lassen sich zu einem manichäisch-türkischen Text zusammenfügen.

1 Vgl. WiLKENS 2000. 
Drei dieser Bruchstücke sind im Katalog der manichäisch-türkischen Texte der Berliner Sammlung von JENS WILKENS ${ }^{2}$ verzeichnet.

Ch/U 7247 (WILKENS 2000, Nr. 4413) + Ch/U 6358 = T. 223, 396a18-b4

Ch/U 7196 (WILKENS 2000, Nr. 4404) = T. 223, 396b16-19

Ch/U 6890 (WILKENS 2000, Nr. 4395) = T. 223, 398a25-b6

$\mathrm{Ch} / \mathrm{U} 7282$ = T. 223, 399c27-400a1.

Die chinesische Buchrolle enthält eine Abschrift des Prajñāpāramitā-Texts T. 223 und umfaßt den Abschnitt von 396a18 - 400a1. ${ }^{6}$ Nur zwei Bruchstücke lassen sich direkt zusammensetzen (Ch/U $6358+\mathrm{Ch} / \mathrm{U}$ 7247), zwischen den übrigen bestehen kleinere oder größere Lücken. Dieser Umstand ist auch der Grund dafür, daß kaum ein zusammenhängender Text im Türkischen greifbar ist. Da das Papier der chinesischen Buchrolle sehr fein ist, scheinen die chinesischen Zeichen durch. Der Lesbarkeit der uigurischen Zeilen tut dies aber keinen Abbruch, da die Schrift recht groß mit starker schwarzer Tusche geschrieben wurde. Es handelt sich um einen halbkursiven Schrifttyp, der dem 10./11. Jh. zuzurechnen ist.

\section{Zur Sprache der Fragmente}

Es finden sich einige Kriterien, die für einen manichäischen Text der älteren Periode (10./11. Jh.) sprechen:

a) Suffixe mit A neben X: Instrumental: $k u s+u n$, aber tuzak+an.

b) Ausgehend vom Akkusativ ätim(ä)zän und der Form sizäy (statt siziฤ) könnte man andere vokallos geschriebene Suffixe ebenfalls mit $A$ lesen.

Transliteration der Fragmente

1. $\mathrm{Ch} / \mathrm{U} 7282$ verso

$01[\quad] \mathrm{yrt}[\quad] \mathrm{k}[\quad] \mathrm{n}$

02 [ ]n qwrtwl m'qyn

03 [ ] 'wykws 'd[ ]w qylync

2 WILKENS 2000.

3 WiLKENS 2000, Nr. 441 vermutet einen „Brief“.

4 Wilkens 2000, Nr. 440 stuft das Fragment als „Brieffragment“ ein.

5 Wilkens 2000, Nr. 439. Der Autor (S. 382, Fußn. 1209) zieht meine frühere Einschätzung des Fragments als Briefrest (BT V, 71 [Nr. 35]) in Zweifel und schreibt bereits: „Vgl. die in M 47 (HandschrReste II) mitgeteilte Legende, in der auch von einem Garten und zwei Brüdern die Rede ist. Allerdings wird dort kein Barhay erwähnt."

6 Die chinesischen Vorderseiten wurden in den drei publizierten Katalogbänden (BT VI, BT XIV und VOHD XII,4) noch nicht aufgenommen. Vier Fragmente (Ch/U 6358, $\mathrm{Ch} / \mathrm{U}$ 6890, Ch/U 7196 und Ch/U 7282) wurden von KUDARA 2000 identifiziert. 
2. Ch/U 6890 verso $^{7}$

$01 \mathrm{t}$ [ ]rym pw "y' [ ]q 'wyz wtwn t'rqm[ ]s

(eine Zeile frei)

02 t'qy ym' tnkry pytykynt[ ]l q'ywr s'pwqr

03 p'rq'nk 'yny sy p'r 'rty : m[ ]l[ myswn 'wlwq

04 [ ]k 'ylyky [ ]ym' m'ny pwrq'nq'

05 [ ]wcy 'rty : ym' 'w[]

06 [ ]r yymyslyk [ ]d ]

3. Ch/U 7196 verso

01 [ ]

02 swv qwty 'ync' t[

03 yrw[ ]wmwzny [

04 []ng [ ]nw[

05 [ ]wt

4. $\mathrm{Ch} / \mathrm{U} 7247+\mathrm{Ch} / 6358$ verso

01 [ ] $[\mathrm{t}]$

02 [ ] y'k q'crwp 'wy[

03 [ ] ] qwswn 'wylwr [

04 [ ]kw twz'q'n q'nw

05 [ ] ] 'tmz pyswrw sw[

06 [ ] ] ]

07 [ ] 'nt' 'wytrw ywrt [ ]

08 [ ]' typ 'wy[]wnty l'r : pyz p [ ]

09 "'yqmz yvl q' mz ywq 'rty [ ]

10 pyrwr 'rtmz 'wykws 'wtswz swvswz [

11 kwyrtkwrty 'nk kyny n'nk svynm'dy [

12 'wylwrty 'tymz 'n yy[ ] q'nm[]n 'y[ ]

13 syz 'nk qwtwnkwzd' [ ] pwl'lm :

14 [ ]w [ ] tynlq qwp [ ]

Transkription und Übersetzung

1. $\mathrm{Ch} / \mathrm{U} 7282$ verso

0101 [ ]ir k[ ]k[ ]n

0202 [ ]n kurtul-mak(1)n

0303 [ ] ükǚ̌ äd[g]ü k1lınč

(umfangreiche Lücke)

7 Das Fragment besteht aus zwei Teilen, die nicht direkt verbunden sind, obwohl es auf dem Foto den Anschein hat. Zwischen der ersten und der zweiten Textzeile beider Teile sind zwei Zeilen frei geblieben, um einen Abstand zwischen zwei Texten zu markieren. Bestätigt wird dies auch vom Textverlauf der chinesischen Zeilen auf der Vorderseite. 
2. $\mathrm{Ch} / \mathrm{U} 6890$ verso

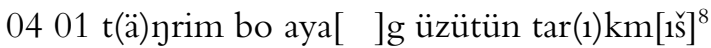

(1 Zeile frei, um zu markieren, daß ein neuer Text(abschnitt) folgt.)

0502 tak1 ymä t(ä) jri bitigint[ä ayu yar]l(1)kayur šapuhr

0603 barxan(a)k (?) ini-si bar ärti : m[ihršah at]l[1]g mešun ulug

0704 [ulušnun]g eligi [ärti :] ymä mani burhanka

0805 [ g]učı ärti : ymä o[1]

0906 [ bi]r yemišlik [et]d[I ]

(umfangreiche Lücke)

\section{3. $\mathrm{Ch} / \mathrm{U} 7196$ verso}

\section{1 [ ]}

1102 suv kutı inčä tep t[edi

1203 ya[r]u[k]umuzni ${ }^{9}$ [

1304 [n] äy '[...]nu[ ]

1405 [ ]ut[ ]

(umfangreiche Lücke)

\section{4. $\mathrm{Ch} / \mathrm{U} 7247+\mathrm{Ch} / \mathrm{U} 6358$}

1501 [ ]t

1602 [ ] yäk kačrup [ ]

1703 [ ]g kušun ölür [ ]

1804 [ äd]gü tuzakan kanu [ ]

1905 [ ]r ät(i)m(ä)z bišuru su[ ]

2006 [ tä]Đrim sizän kutuyuzda

2107 [ ] : anta ötrü yurt [

2208 [in]čä tep ötünti-lär : biz b[arča ]

2309 ay(1)g(1)m(1)z y(a)vlak(1)m(1)z yok ärti [

2410 berür ärt(i)m(i) z üküš otsuz suvsuz [yerlärig]

2511 körtgürti äy keni näy s(ä)vinmädi [

2612 ölürti ätim(ä)zän ye[p ] kan(1)m[1z1]n ič[

2713 sizäy kutuyuzda [] bolal(1)m :

2814 [ ]u [ ] tinl(1)g kop [ ]

\section{Übersetzung}

Wegen der unvollständigen Zeilen und der Lücken ist nur teilweise ein Verständnis des Textes zu erlangen. Hier lege ich einen ersten Versuch vor. Die textfreie Zeile zwischen den Zeilen 04 und 05 deutet darauf hin, daß es sich um eine Sammlung von Texten handelt. Ob alle Abschnitte zu einer möglichen Kirchengeschichte wie der zweite gehören, ist ungewiß.

8 WiLKENS 2000, Nr. 439: tetsi.

9 Leider ist die Lesung, so auch bei WILKENS 2000, Nr. 440, nicht sicher, man könnte auch ya[z]uk 'Sünde' vorschlagen. 
1. Ein erster Abschnitt umfaßt die Zeilen 01-04, wo sicher nur zwei Reste zu deuten sind: [...] durch Befreitwerden [...] viele gute Taten [...]

Auf eine umfangreiche Lücke folgt die Zeile 04, die das Ende eines (des ersten?) Sinnabschnitts zu sein scheint:

'[...] mein Gott. Mit dieser schlech[t-...]en Seele entfernte er sich (?).'

2. Der zweite Abschnitt umfaßt die Zeilen 05-09. Hier liegt der Anfang einer Bekehrungsgeschichte vor, die auf einer parthischen Vorlage basiert. ${ }^{10}$

'Und ferner geruht er in seinem göttlichen Buch [zu sagen]: Der Herrscher (?) Šapuhr hatte einen jüngeren Bruder, namens M[ihršah. Er war] König des großen [Reich]es Mesene. Und er war [feindlich] gegen Mani Burhan. Und jener [legte] einen Garten [an, ...].'

3. Von einem dritten Abschnitt nach einer umfangreichen Lücke sind nur zwei Segmente zu übersetzen:

'(11) Die Majestät des Wassers sprach so [...] (12) unsere Lichter [...]'

4. Der vierte und letzte Abschnitt (Zeilen 15 bis 28) bietet ein etwas längeres Stück, allerdings ohne eine vollständige Zeile, so daß wiederum das Verständnis sehr erschwert ist. Auch hierzu ein erster Versuch:

'(15) [...] den Dämon in die Flucht schlagend [...] (16) [...] mit (?) Vögeln töte[nd ...] (17) [...] mit [gut]en Fallen zufrieden seiend [...] (18) [...] unser Fleisch kochend $[\ldots]$ (19) [...] mein Gott, bei Eurer Majestät [...].

(21-27) Darauf geruhten die [im] Land ${ }^{11}$ (?) [seienden Menschen (?)] zu bitten: ,Wir a[lle ...] hatten keine schlechten und üblen Taten. [Alle Arten von Almosen (?)] hatten wir gegeben. Viel pflanzenlose und wasserlose [Länder (?) ...] zeigte er (der Dämon?). Ganz am Ende (?) freute er (der Dämon?) sich gar nicht. [Viele ...] tötete er. Unser Fleisch aß er, unser Blut trank er. [Jetzt ...] wollen wir bei Eurer Majestät [gerettet (?) ...] sein!' [...] (28) [...] Lebewesen, alle [...].'

\section{Bemerkungen zu einzelnen Passagen und Wörtern}

05-09 Dieser Passus basiert auf einer parthisch überlieferten Bekehrungsgeschichte, über die WERNER SUNDERMANN (BT XI) schreibt: „Daß mindestens mit einer hagiographischen Stilisierung der manichäischen kirchengeschichtlichen Überlieferung gerechnet werden muß, bedarf keiner Ausführung. Selten aber wird diese Formung der Tradition durch Ritus und Lehre so deutlich wie in der hier erzählten Geschichte von der Bekehrung des Mihršāh, deren historischer Charakter allerdings anzuzweifeln ist: Mani wünscht dem Mihršāh Frieden (/V/1), er erweckt ihn aus seiner Bewußtlosigkeit durch Handauflegung zu der neuen Existenz eines gläubigen Anhängers

10 Vgl. BT XI, S. 102.

11 Zur semantischen Entwicklung von yurt 'Zelt, Wohnort, Land' etc. vgl. ED 958. 
(...) “12. Die Erzählung spielt in der Landschaft Mesene im Zweistromland, die auch in der Manibiographie des Kölner Mani-Kodex eine Rolle spielt. Nach dem Kölner Kodex ist Manis Aufenthalt in der Mesene nicht mit einer Begegnung mit einem Herrscher, sondern mit den Täufern verbunden gewesen. ${ }^{13}$ Der parthischen Bezeichnung myšswn, die genauso auch in die altuigurische Version übernommen wurde, stehen syrisch Maišān und griechisch Maïoavoí gegenüber.

05 t(ä)yri bitigint [ä] 'in seinem göttlichen Buch'. Bisher ist kein t(ä)yri bitigi 'Gottes Buch' aufgetaucht, deshalb neige ich hier dazu, das Wort t(ä)yri als ein Epitheton 'göttlich' aufzufassen. Um welches Buch es sich hier handelt, kann nicht mit Sicherheit gesagt werden. Wenn man jedoch an eine kirchengeschichtliche Darstellung mit Bekehrungsgeschichten denkt, dann ist der Bezug auf Mani selbst gegeben.

05-07 Die mögliche parthische Vorlage nach BT XI 10.6-8 lautet:

'Ferner hatte der König der Könige Šābuhr einen Bruder, den Herrn der Mesene, und er hieß Mihršāh.'

byd šbwhršsh'n šh br'd bwd (m)yšwn xwd'y u myhr šh n'm

šapuhr barxan(a)k ini-si bar ärti : m[ihršah at]1[1]g mešun ulug [ulušnun]g eligi [ärti :]

Bis auf Kleinigkeiten ist die Übereinstimmung perfekt. So fehlt im Uigurischen eine Entsprechung furr parth. byd am Anfang des Satzes. Der Uigure muß sich entscheiden für 'jüngeren' oder 'älteren' Bruder, im Parth. allgemein 'Bruder'. Dem Landschaftsnamen Mesene (parth. myšwn) wird im Uig. 'großes [Reich]' nachgesetzt. Als Äquivalent für parth. $x w d$ 'y dient altuig. elig 'König'.

$06 p^{\prime} r q^{\prime \prime} k$. Hinter diesem Wort, dessen Lesung vielleicht barkay ${ }^{14}$ gewesen sein könnte, muß sich ein Titel eines Herrschers oder Königs verbergen, wie auch YUTAKA YOSHIDA in einem Email meinte. Hier möchte ich zwei sehr unterschiedliche Lösungen vorstellen, die aber allenfalls Annäherungen sein können. Zum einen denke ich an das mittelpersische Wort frhng 'education, knowledge ${ }^{15}$. STEINGASS fügt auch andere Bedeutungen hinzu wie 'greatness; excellence' ${ }^{16}$, und so könnte es sich um ein Substitut für alttürkisch kut 'Würde, Majestät' handeln, oft in Königstiteln verwendet, ganz besonders in dem Titel der Könige von Qočo: ıdukkut 'Heilige Majestät'. Man muß sich aber fragen, warum der altuigurische Autor nicht den persischen Titel šāhānšăh, vielleicht auch pādšăh nach einer anderen Überlieferung, übernommen hat.

12 SUNDERMANN 1981, S. 101-102.

13 RÖMER 1994, S. 105-111 (und folgende Seiten über Manis Aufenthalt in der Mesene). Hier wird auch weitere Literatur über die Mesene genannt und nach relevanten Aussagen bezüglich Manis Aufenthalt in derselben beleuchtet.

14 Wie erwähnt, las Wilkens 2000, Nr. 439, Barhay.

15 DURKIN-MEISTERERNST 2004, S. 155a.

16 STEINGASS S. 926a. 
Und hier wäre eventuell die zweite Lösung: Ein ursprünglich in manichäischer Schrift geschriebenes $p^{\prime} d x^{\prime} y^{\prime} \underline{h}^{17}$ wurde unvollkommen in die uigurische Schrift transponiert, eben in der obigen Form $p^{\prime} r q^{\prime \prime} k$. Dabei wären gravierende Mißinterpretationen anzunehmen: manichäisches $d$ wurde als $r$ gelesen, šy' als " oder 'n etc., was eine geringe Kenntnis der manichäischen Schrift voraussetzen würde. Da jedoch dann nur in diesem Wort solche Fehler vorkämen, plädiere ich zur Zeit eher für die erstere Deutung.

06 Zum Herrn der Mesene, Mihršah, vgl. die Ausführungen in BT XI, p. $102 \mathrm{Fußn}$. 2. WERNER SUNDERMANN hält die Geschichtlichkeit dieses Königs für fraglich.

07-08 Parthisch BT XI.10.8-9

'Und (gegen) die Religion des Apostels war er sehr feindlich gesinnt.'

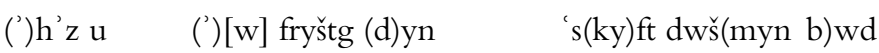

ymä mani burhanka [ g]učı ärti :

Der Uigure hat fryštg dyn nicht als 'Religion des Apostels' verstanden, sondern als mani burhan 'Mani Buddha', einer Bezeichnung, die eher auf die Interpretation des Kompositums als 'Apostel der Religion' weist. Daher wäre es vielleicht auch möglich, die parthische Konstruktion als 'Apostel der Religion' zu verstehen. Dem steht jedoch entgegen, daß üblicherweise zwischen den Nomina ein izafetisches $c y$ stehen sollte. ${ }^{18}$

08-09 Parthisch BT XI.10.9-10

'Und einen Garten hatte er angelegt'

(')wš bwdyst'n wy $\quad$ 'r' $\left.{ }^{\prime}\right)[\check{s}] t$ ' $y w$

ymä ol [...] [bi]r yemišlik [et]d[i]

Die Wortfolge ist zwischen beiden Sprachen etwas anders, so fällt auf, daß 'ein' am Ende des parthischen Satzes steht. Des weiteren muß vor [bi]r yemišlik, dem Äquivalent für bwdyst'n '(Obst)-Garten'19, noch eine größere Ergänzung angenommen werden, für die es im parthischen Satz keine Vorlage gibt, es sei denn, daß der im Nachsatz folgende Gedanke 'Er ist sehr herrlich und außerordentlich weit, kein anderer ist ihm ebenbürtig.' in der Lücke Platz gefunden hätte.

11 suv kutı 'Majestät des Wassers'. Vgl. M II 12,3: yer suv kutı irinür 'Die Majestät der Erde (Erde und Wasser) weint'.

16 kačrup. Von einer kontrahierten Form von kačur- 'fliehen lassen, in die Flucht schlagen' vermute ich hier das entsprechende Konverb. Bei Maḥmūd al-Kāšyarī ist im Falle von kačur- der Vokalismus voll erhalten, doch weisen

17 DURKIN-MEISTERERnSt 2004, S. 258a.

18 Freundlicher Hinweis von DESMOND DURKIN-MEISTERERNST.

19 DURKIN-MEISTERERNST 2004, S. 116a. 
andere Ableitungen wie kačruš- und kačrumsin- ebenfalls auf die Erscheinung hin. ${ }^{20}$

17 kušun (Instrumental von kuš 'Vogel, Vögel') 'mit Vögeln', dies könnte vielleicht als ein Hinweis auf die Falkenjagd gedeutet werden.

18 tuzak 'Falle' (ED 573b). Zwar liegt in BT XI 13 eine Erzählung vor, in welcher von einem Jäger und seinen Fallen und Schlingen die Rede ist, doch läßt sich kein direkter Anknüpfungspunkt herausschälen.

21-27 Dieser Passus ist wohl eine Klage der durch Dämonen gepeinigten Menschen, die befreit werden wollen. Als besonders schrecklich und verwerflich wird die Absicht des Dämons (?) dargestellt, das Fleisch der Menschen zu essen und ihr Blut zu trinken.

25 äy keni 'spätestens' (?).

26 ätim(ä)zän ye[p ] kan(i)m[izl]n ič[ti (?)] 'Unser Fleisch aß er, unser Blut trank er.' In den Kephalaia 80 heißt es über die Gebote für die Electi: „Das Dritte ist die Reinhaltung des Mundes. Er soll seinen Mund von allem Fleisch und Blut reinhalten. ${ }^{\text {21 }}$ Hier geht es aber nicht um Electi, sondern offenbar um Hörer, die von Dämonen bedrängt werden. Im Mythos wird ausgeführt: „Als dann der ,erste Mensch “ und die ,Weibliche der Glorien', der erste Mann und die erste Frau, über die Erde zu herrschen begannen, da erwachte in ihnen die $\bar{A} z$. Und Raserei durchdrang sie, und sie begannen Quellen zuzuschütten, Bäume und Pflanzen zu schlagen, in großer Raserei über die Erde zu herrschen und gierig zu werden, Und vor den Göttern fürchten sie sich nicht. Und jene fünf Elemente, aus denen die Welt errichtet ist, erkennen sie nicht, sondern quälen sie ohne Unterlaß." ${ }^{22}$ In den Sündenbekenntnissen werden diese Quälereien der bedrängten Seele thematisiert. Aber in obiger Passage liegt wohl doch ein anderer Sachverhalt zugrunde, denn 'Fleisch' und 'Blut' sind personal markiert, weshalb ich, wie oben schon angedeutet, annehme, daß der Text von der Bedrängung durch Dämonen, wenn im vorhandenen Text auch nicht explizit ausgedrückt, spricht.

\section{II.}

\section{Die Fragmente $U$ 6 a-d $\left(D \text { I } H^{I}\right)^{23}$}

$\mathrm{Ob}$ die nun folgenden Textreste von $\mathrm{U} 6 \mathrm{a}-\mathrm{d}$ als eine Bekehrungsgeschichte interpretiert werden können, bleibt etwas fraglich. Bisher ist für diese Erzählung noch kein manichäischer Bezug vermutet worden. Aus AlBERT GRÜN-

20 ED S. 592-593.

21 Nach BÖHLIG 1980, S. 192.

22 BÖHLig 1980, S. 117-118.

23 LE COQ 1919, S. 101 schreibt: T.I. H ${ }^{\mathrm{I}}$. Nach dem Eintrag auf dem Papier ist beides möglich. Es ist eindeutig, daß das Bruchstück während der ersten Campagne in D (= Dakianusšähri $=$ Qočo) gefunden wurde. 
WEDELs Beschreibung haben wir zwar einen generellen Eindruck über den Bau $\mathrm{H}^{\mathrm{I}}$ in Qočo (Idikutšähri) ${ }^{24}$, doch abgesehen von Resten einer sanskritischen Palmblatthandschrift, erwähnt er nichts an Manuskripten. Eine religiöse Zuordnung des Tempels wurde auch nicht vorgenommen.

Wegen des Wortes mogoč 'Magier' ${ }^{25}$ lag es nahe, zunächst an christliche Herkunft zu denken. So schreibt AlBert VON LE COQ: „Zerstörtes Doppelblatt aus einem christlichen Buch (Seitenbreite $6 \mathrm{~cm}$, Höhe unbekannt). Gefunden in Ruine $\mathrm{H}^{\mathrm{I}}$ in Chotscho. Der Inhalt ist ein Teil einer anderen Handschrift der von F.W.K. MÜLLER in "Uigurica« veröffentlichten Apokryphe "Die Anbetung der Magier«. Die Schrift ist eine sonst nur von Manichäern, nie von Buddhisten benutzte sehr kleine Variante der spätsoghdischen Schrift und zeigt Eigenheiten der unter den Manichäern üblichen Schreibweise. “26 Er erwähnt nicht die dazugehörigen Bruchstücke $\mathrm{b}$ bis $\mathrm{d}$, die richtig einzuordnen ohne Identifizierung unmöglich ist. Von dem größten Bruchstück (a) hat LE COQ eine Seite umschrieben.

Albert vON LE COQs Hinweis auf die übliche Schreibweise der Manichäer sowie der ergänzbare Ausdruck [tän]gri mani [burhan] im Bruchstück c4 lassen den Schluß zu, daß die Fragmente dieser Handschrift eher Reste eines manichäischen Textes sind. Vielleicht läßt die Wendung šanšai üdintä 'vor dem König' den Schluß zu, daß es sich um eine der Bekehrungsgeschichten handeln könnte. Um diese Annahme eindeutig beweisen zu können, ist der erhaltene Textbestand nicht ausreichend genug.

\section{Bruchstück a Seite I}

(recto)

01 kılınčl(1)g üzütlär kim

02 kök t(ä) yri-dän anx(a)rw(a)z(a)n

03 -tan sars1-g ämgäkdä

24 GRÜNWEDEL 1905, S. 21-22.

25 BANG 1926, S. 44 Fußn. 2. Das auf sogd. mury št zurückgehende Wort ist aber nicht auf eine Religion festgelegt, vgl. Belege in manichäisch-türkischen Texten: M III Nr. 22 (U 63, in manichäischer Schrift) r 4 mogoč nomın 'die Magier-Lehre' könnte sich auf den Zarathustrismus beziehen (vgl. auch Wilkens 2000, Nr. 51, S. 78, Fußn. 186). U $153 \mathrm{~b}$ [manichäische Schrift] verso (?) 5 murwc = mogoč (ohne Kontext); TM 509b [verlorenes Fragment in manichäischer Schrift] verso (?) 2: m[ogo]čll]ar. Zur Herkunft des Wortes: AlOÏS VAN TONGERLOO 1992, S. 70, leitet es von einem nicht belegten sog-

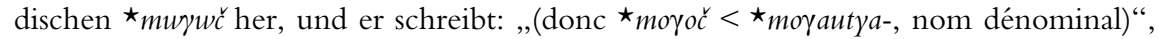
aber das von ILYA GERSHEVITCH in GMS $₫ 1080$ behandelte ist ein deverbales Suffix zur Bildung von Abstracta. Schon 1988 diskutierte N. Sims-Williams das Wort 'magus' im Sogdischen, und bezüglich des altuigurischen Belegs in der Erzählung über die Drei Magier schreibt er: ,which might derive from a Sogdian plural such as $\star_{m \gamma w s t}$ or $\star_{m w \gamma y}{ }^{2} t$ (with vowel assimilation, replacement of the non-Turkish sequence $s t$ by c). “ Ich denke, daß die ältere Erklärung einleuchtender ist.

26 Le CoQ 1919, S. 101. 
04 t(ä)zär inär bo yerd[ä]

05 [ t]uml-1g[

06 [ ]yan[ ]

(verso)

01 ol kudug agzintak1

02 ül-gü ot $\mathrm{k}(\ddot{a}) n t \ddot{~ o l}$

03 ät'özlüg kiši-lärn(i)n

04 [is]ig özi kö-ki

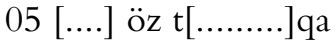

$06[\ldots] \mathrm{ug}[\quad]$

\section{Übersetzung}

(recto)

Die Seelen mit [schlechten] Taten sind es, die vor den vom Himmel und Zodiakus $^{27}$ (kommenden) schrecklichen Qualen fliehen und hinabsteigen. Auf dieser Erde $(\ldots)[\mathrm{k}]$ alt $(\ldots)^{28}$

(verso)

Ein Maß (?) $)^{29}$ Feuer am Rande des Brunnens ${ }^{30}$ ist es, das das Leben der einen Körper habenden Menschen (...)

Bruchstück a Seite II

(recto)

01 bizäy ötüg

02 tijlap ol ataš ${ }^{31}$

03 tutzun tep

04 yarl(1)kasar : an[...]

05 [ ]an[ ] üzä

$27 \mathrm{Zu}$ den Belegen des Wortes $a n x(a) r w(a) z(a) n<$ sogd. 'nxrwzn, 'xrwzn 'Zodiak' vgl. UW 139b.

28 JeNS WiLkens erwägt, ob in diesen Zeilen an die an den Zodiak gefesselten Dämonen erinnert wird, wie es auch im Gigantenbuch elaboriert wird.

29 Das ülgü gelesene Wort bereitet große Schwierigkeiten, soll man an eine Ableitung von öl- 'sterben' denken? Was aber ist das '(zu) sterbende Feuer'? Selbst wenn man wieder an die christliche Magiererzählung denkt, in der es heißt, daß sich aus dem Brunnen ein mächtiges Feuer erhoben hat (ol kudug ičintä bir korkınčg ulug yaruk oot yalın birlä ünüp), bleibt die Stelle im dunkeln.

30 kudug agzıntakı 'am Rand des Brunnens befindlich', dieser Beleg für agız fehlt in UW. Wie im Türkeitürkischen kann man auch mit der Bedeutung 'Rand' rechnen, obwohl natürlich auch 'Öffnung' gemeint sein kann.

31 UW 258 b: statt ataš sei antag zu lesen. Zwar sind die finalen Formen $-q$ und $-s$ sehr ähnlich, doch vom Kontext her möchte ich der ersteren und älteren Lesung den Vorzug geben. 


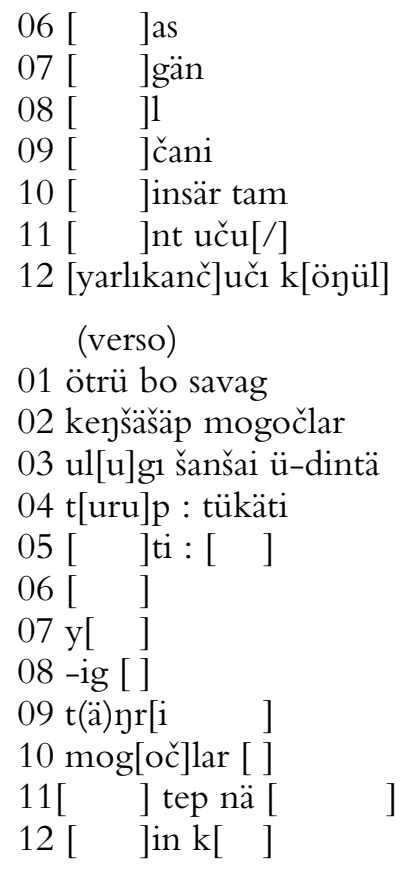

Übersetzung

(recto)

Wenn er geruht zu befehlen, daß er (?), nachdem er unsere Bitte gehört hat, (mich?) als Freund (?) behalten solle, (...)

(verso)

Nachdem sie sich über diese Rede beraten hatten, stand (?) der Älteste der Magier $^{32}$ vor dem König ${ }^{33}$, vollständig ..., Gott ..., die Magier (...)

Bruchstück b

(recto?)

01 [ ]

02 [ ]lämäk ${ }^{34}[$ ]

03 -mak : ymä []

04 tü-pintäki [ ]

32 mogoclar ulugı 'Ältester der Magier': wer könnte damit gemeint sein? JENS WILKENS weist auf die Möglichkeit hin, daß man hier vielleicht den sasanidischen Mōbed Kerdīr sehen könnte (ich danke ihm für diesen Gedanken). Vgl. auch SimS-WiLliams 1990.

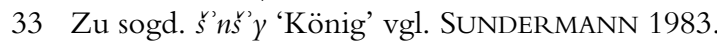

34 Der Vergleich mit ölmäk in Z. 6 zeigt, daß hier ein anderer Infinitiv eines Verbs vorliegen muß, das auf -lä endet. 
05 [e]l-än k(ä)ntü [ ] ]

06 öl-mäk küni '[ ]

(verso?)

$01\left[\begin{array}{ll}{[} & ] 1\end{array}\right]$

$02[\quad] n l(1) g$ šs $[\mathrm{a}] \mathrm{tu}$

03 [ ] 1 kim anx(a)rw(a)z(a)n

04 [ ]ar kor yas

05 [ ] kodgu ag-1

06 [ ]si-lär üzä

Übersetzung

(recto?)

[...] Und die am Boden [des ...] befindlichen [...], [herr]sche! Selbst [am] Tage des Todes $[\ldots]$

(verso?)

[...] die [...] Leiter [...], die [zum (?)] Zodiakus [...] Schaden und Verlust [verursachende] abzulegende Güter [...]

Bruchstück c

$$
\begin{aligned}
& \text { (recto?) } \\
& 01 \text { [ } \\
& 02 \text { ü-dintä [ ] ] } \\
& 02 \text { ara surč[ ] } \\
& 03 \text { uvutlug [ ] } \\
& 04 \text { inčä bälg[ür ] } \\
& 05 / / \text { lay[ } \\
& \text { (verso?) } \\
& 01 \text { [ ]1[ ]n } \\
& 02[\text { [ ] b(ä)lgürgäy } \\
& 03 \text { [ ] üd-ün } \\
& 04 \text { [ tn]gri man-i } \\
& 05 \text { [ ]sangay } \\
& 06[\text { ]inč }
\end{aligned}
$$

\section{Übersetzung}

$$
\text { (recto?) }
$$

$[\ldots]$ zur Zeit $[$ des $\ldots$.$] zwischen [\ldots]$ stolp $\left[\operatorname{ernd}(?)^{35} \quad \ldots\right]$ schamvoll $[\ldots]$ so erschei[nend ...]

35 Die Lesung von swrc[...] bleibt unklar, es gibt nur ein Verb sürč- 'to stumble' (ED 845), das eventuell auch hier zugrunde liegt. 
(verso?)

$[\ldots]$ wird erscheinen, $[\ldots]$ zur Zeit $[\ldots]$ göttlicher $\operatorname{Mani}^{36}[\ldots]$ wird $[\ldots]$

Bruchstück d

(recto?)

01[

$02 \mathrm{ym}[\ddot{a}$

$03 \mathrm{~s}[\mathrm{]n}[$

04 künl-i [

05 tün $\mathrm{t}[$

$06[\ldots]$ si nng [ ]

$07[.$. bo]šuyur [ ]

(verso?)

01[

02[

$03[$ ]ča

04 [ ] ärür

05 [ ] öl-ürgüči

06 [ ]n yaya [ ]

07 [ k1]l-1nč[ ]

08 [

Übersetzung

(recto?)

[...] Un[d ...] [Nacht und] Tag [...] Nacht [...] [fre]i kommt ${ }^{37}[\ldots]$

(verso?)

$[\ldots]$ ist. $[\ldots]$ tötend $[\ldots]$ Elefant $[\ldots][\mathrm{T}]$ at $[\ldots]$

\section{Bibliographie}

BANG, WILLI (1926): Türkische Bruchstücke einer nestorianischen Georgspassion. In: Le Muséon 29, S. 41-75.

Böhlig, Alexander (unter Mitarbeit von) Jes P. Asmussen (1980): Die Gnosis. Dritter Band. Der Manichäismus, Zürich/München.

Clauson, Gerard (1972): An Etymological Dictionary of Pre-Thirteenth-Century Turkish, Oxford.

36 In anderem Zusammenhang wird Mani auch yaruk täyri mani burhan 'Lichtvoller, Göttlicher Mani Buddha' genannt, vgl. Hamilton 1986, Text 5.1.

37 Die Emendation zu [bo]šu- 'frei kommen, werden' stellt nur eine Möglichkeit dar. 
Doerfer, Gerhard (1974): Die Literatur der Türken Südsibiriens. In: Philologiae Turcicae Fundamenta II, Wiesbaden, S. 866 ff.

Durkin-Meisterernst, Desmond (2004): Dictionary of Manichaean Middle Persian and Parthian, Turnhout.

GersheVitch, Ilya (1954): A Grammar of Manichean Sogdian, Oxford.

GHARIB, B. (1995): Sogdian Dictionary, Tehran.

GRÜNWEDEL, AlBERT (1905): Bericht über archäologische Arbeiten in Idikutschari und Umgebung im Winter 1902-1903, München.

Hamilton, James Russell (1986): Manuscrits ouïgours du IX $X^{e}-X^{e}$ siècle de TouenHouang, I-II, Paris.

KudAra, Kōgi (2000): A General Catalogue of the Chinese Texts from East Turkestan in the Berlin Collection (provisional trial edition), Kyoto.

KudARA, KōGI (2005): Chinese Buddhist Texts from the Berlin Turfan Collections Volume 3, hrsg. von T. HASUIKE / M. MitANI, Stuttgart (Verzeichnis der Orientalischen Handschriften in Deutschland XII, 4).

Le CoQ, Albert von (1909): Kurze Einführung in die uigurische Schriftkunde. In: Mitteilungen des Seminars für Orientalische Sprachen an der Friedrich-WilhelmsUniversität in Berlin, Westasiatische Studien 11, S. 93-109.

RÖHRBORN, KlAUS (1977-): Uigurisches Wörterbuch. Sprachmaterial der vorislamischen türkischen Texte aus Zentralasien, I-VI, Wiesbaden.

RÖMER, Claudia E. (1994): Manis frühe Missionsreisen nach der Kölner Manibiographie. Textkritischer Kommentar und Erläuterungen zu p. 121 - p. 192 des Kölner ManiKodex, Opladen.

SCHMITT, GERHARD / THOMAS THILO (1975): Katalog chinesischer buddhistischer Textfragmente Band 1. In Zusammenarbeit mit I. INOKUCHI, Berlin (Berliner Turfantexte VI).

Sims-Williams, Nicholas (1988): Syro-Sogdica III: Syriac elements in Sogdian. In: A green leaf, Papers in honour of Professor Jes P. Asmussen, Leiden (Acta Iranica 28), S. 145-156.

Sims-Williams, Nicholas (1992): The Sogdian fragments of Leningrad II: Mani at the court of the Shahanshah. In: In honor of Richard Nelson Frye. Aspects of Iranian Culture. Bulletin of the Asia Institute 4 (1990), S. 281-288.

Steingass, Francis JosePh (1892): A Comprehensive Persian-English Dictionary, London.

SUNDERMANN, WERNER (1981): Mitteliranische manichäische Texte kirchengeschichtlichen Inhalts, Berlin (Berliner Turfantexte XI).

Sundermann, Werner (1983): Soghdisch šnšy. In: Altorientalische Forschungen 10, S. 193-195.

Sundermann, Werner (2001): Manichaica Iranica. Ausgewählte Schriften von Werner Sundermann, hrsg. von CHRistiane ReCK, DieTER WeBER, Claudia LeURini, ANTONiO PANAINO, 1-2, Roma (Serie Orientale Roma LXXXV, 1-2).

Thilo, Thomas (Hrsg.) (1985): Katalog chinesischer buddhistischer Textfragmente Band 2, Berlin (Berliner Turfantexte XIV). 
TongerloO, Aloïs van (1992): Ecce Magi ab oriente venerunt. In: Acta Orientalia Belgica VII, S. 57-74.

Wilkens, Jens (2000): Manichäisch-türkische Texte der Berliner Turfansammlung, Stuttgart

(Verzeichnis der Orientalischen Handschriften in Deutschland XIII, 16).

ZIEME, PeTER (1975): Manichäisch-türkische Texte, Berlin (Berliner Turfantexte V). 


\section{Abbildungen $^{38}$}
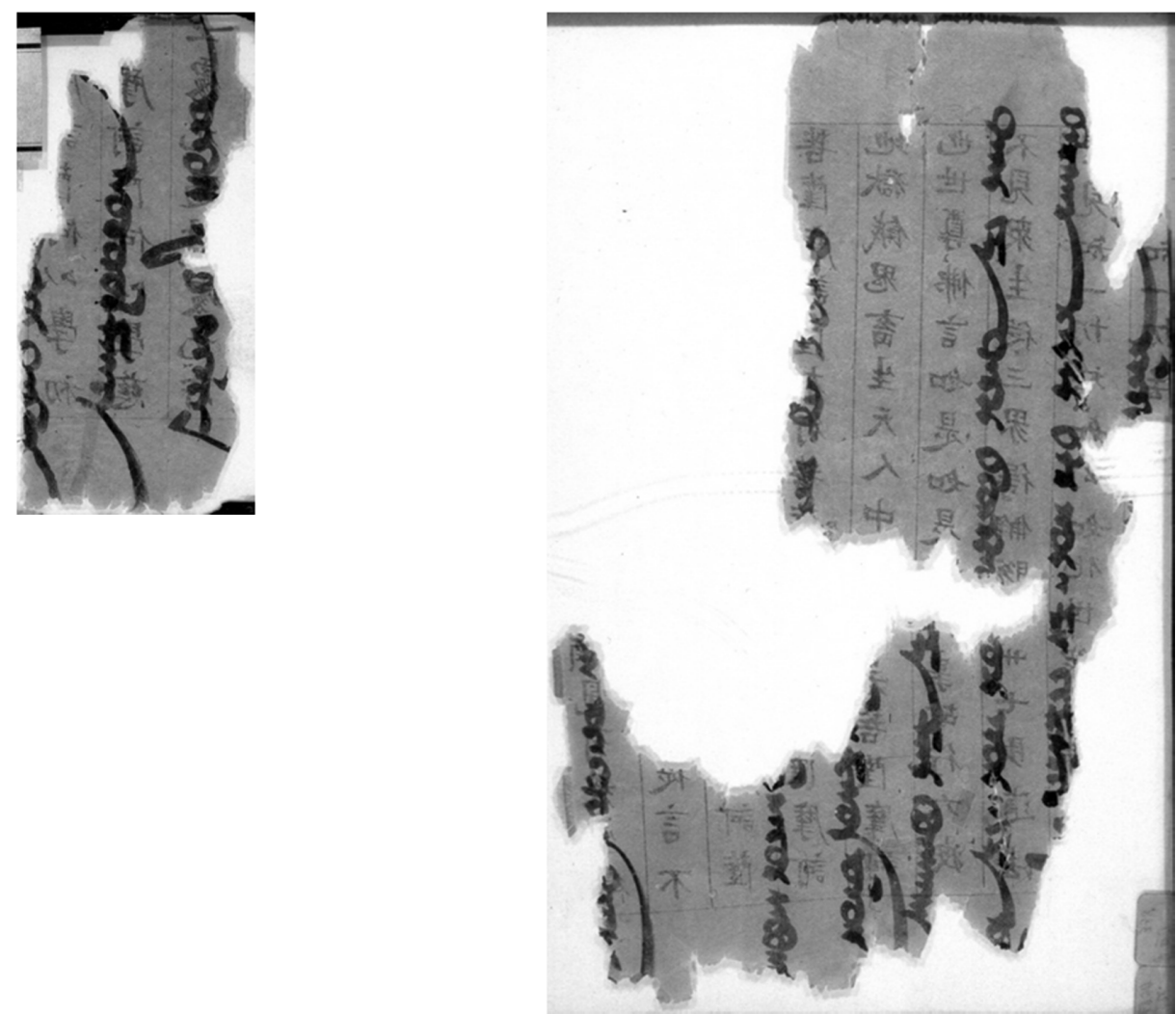

Abb. 1: Ch/U 7282 verso

Abb. 2: Ch/U 6890 verso

38 Da zwischen den einzelnen Teilen der Handschrift große Lücken bestehen, werden die Fragmente bis auf die direkt zusammensetzbaren in Abb. 4 einzeln wiedergegeben. 


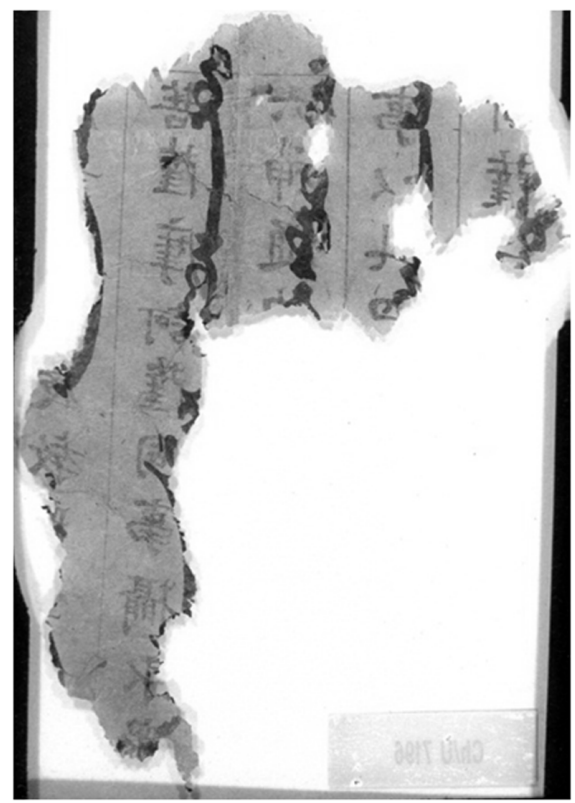

Abb. 3: Ch/U 7196 verso

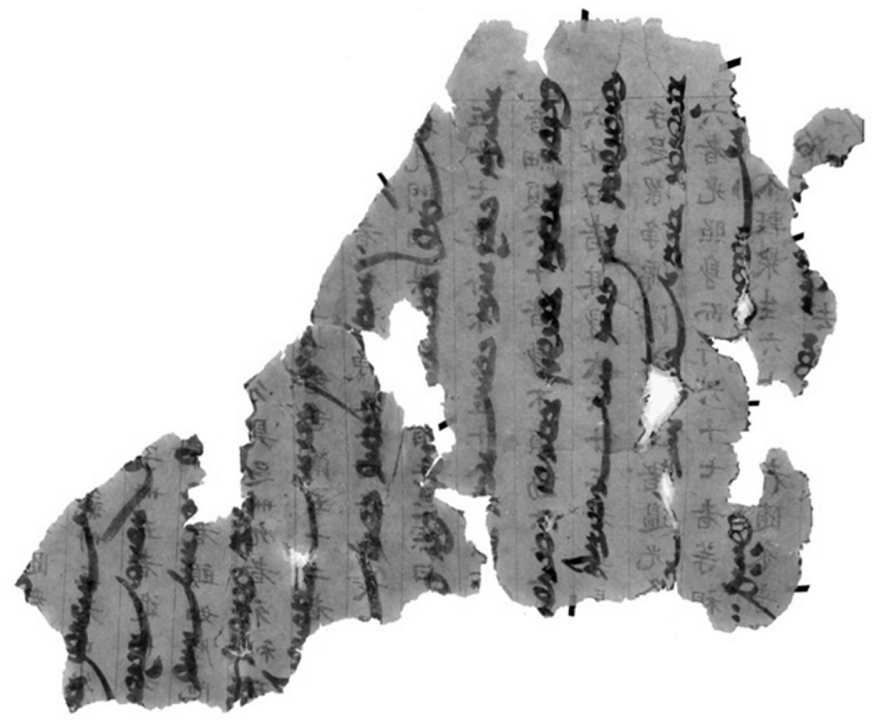

Abb. 4: Ch/U $7247+\mathrm{Ch} / \mathrm{U} 6358$ verso 


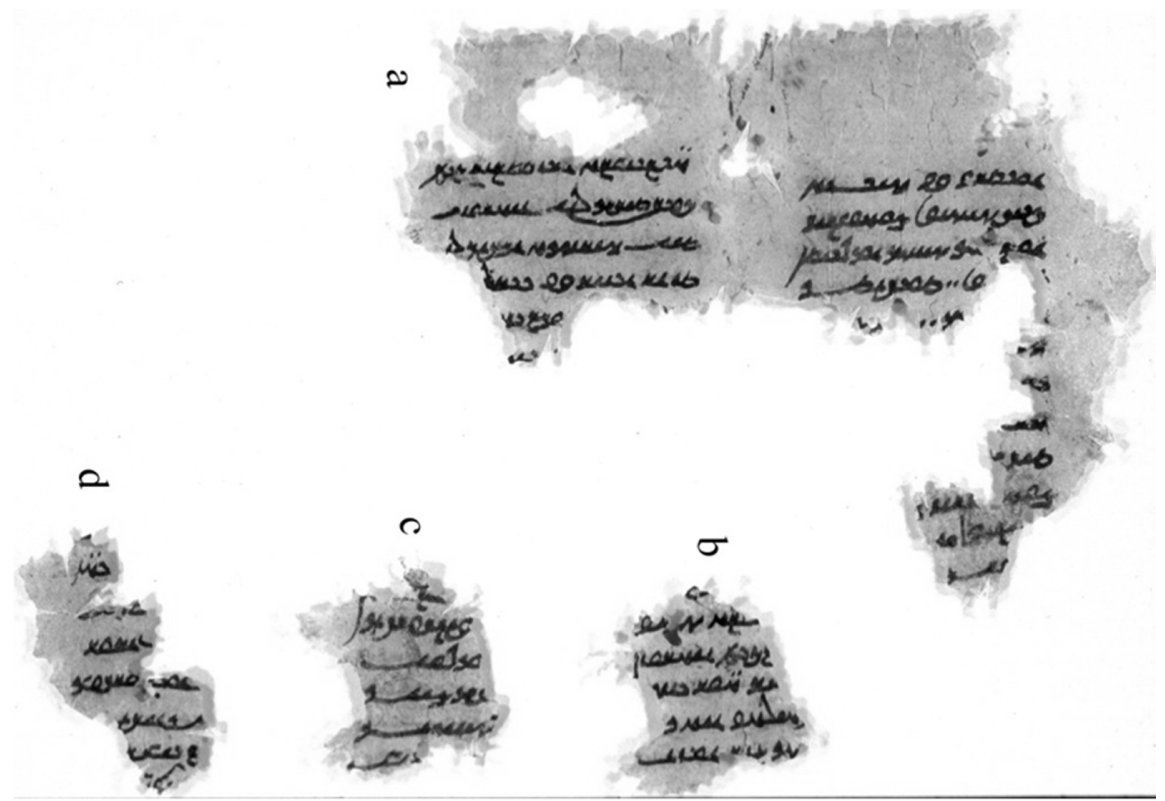

Abb. 5: U 6 a I recto, II verso, $\mathrm{U} 6 \mathrm{~b}$ verso, $\mathrm{U} 6$ c verso, $\mathrm{U} 6 \mathrm{~d}$ verso (D I HI)

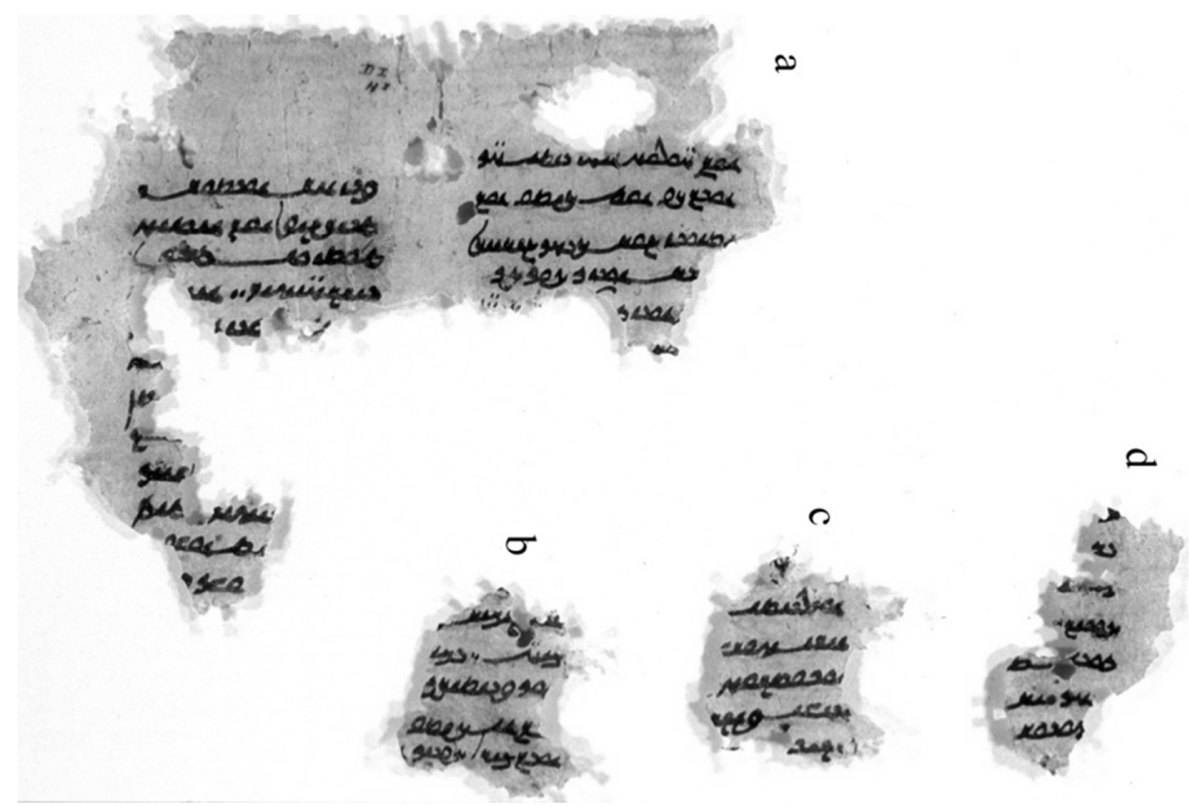

Abb. 6: $\mathrm{U} 6$ a I verso, II recto, $\mathrm{U} 6 \mathrm{~b}$ recto, $\mathrm{U} 6 \mathrm{c}$ recto, $\mathrm{U} 6 \mathrm{~d}$ recto (D I HI) 